\title{
Random forest estimation of genomic breeding values for disease susceptibility over different disease incidences and genomic architectures in simulated cow calibration groups
}

\author{
S. Naderi, T. Yin, and S. König ${ }^{1}$ \\ Department of Animal Breeding, University of Kassel, 37213 Witzenhausen, Germany
}

\begin{abstract}
A simulation study was conducted to investigate the performance of random forest $(\mathrm{RF})$ and genomic BLUP (GBLUP) for genomic predictions of binary disease traits based on cow calibration groups. Training and testing sets were modified in different scenarios according to disease incidence, the quantitative-genetic background of the trait $\left(\mathrm{h}^{2}=0.30\right.$ and $\left.\mathrm{h}^{2}=0.10\right)$, and the genomic architecture [725 quantitative trait loci (QTL) and 290 QTL, populations with high and low levels of linkage disequilibrium (LD)]. For all scenarios, 10,005 SNP (depicting a low-density 10K SNP chip) and 50,025 SNP (depicting a 50K SNP chip) were evenly spaced along 29 chromosomes. Training and testing sets included 20,000 cows (4,000 sick, 16,000 healthy, disease incidence $20 \%$ ) from the last 2 generations. Initially, 4,000 sick cows were assigned to the testing set, and the remaining 16,000 healthy cows represented the training set. In the ongoing allocation schemes, the number of sick cows in the training set increased stepwise by moving $10 \%$ of the sick animals from the testing set to the training set, and vice versa. The size of the training and testing sets was kept constant. Evaluation criteria for both GBLUP and RF were the correlations between genomic breeding values and true breeding values (prediction accuracy), and the area under the receiving operating characteristic curve (AUROC). Prediction accuracy and AUROC increased for both methods and all scenarios as increasing percentages of sick cows were allocated to the training set. Highest prediction accuracies were observed for disease incidences in training sets that reflected the population disease incidence of 0.20 . For this allocation scheme, the largest prediction accuracies of 0.53 for $\mathrm{RF}$ and of 0.51 for GBLUP, and the largest AUROC of 0.66 for RF and of 0.64 for GBLUP, were achieved using 50,025
\end{abstract}

\footnotetext{
Received January 12, 2016.

Accepted May 23, 2016.

${ }^{1}$ Corresponding author: sven.koenig@uni-kassel.de
}

SNP, a heritability of 0.30 , and 725 QTL. Heritability decreases from 0.30 to 0.10 and QTL reduction from 725 to 290 were associated with decreasing prediction accuracy and decreasing AUROC for all scenarios. This decrease was more pronounced for RF. Also, the increase of LD had stronger effect on RF results than on GBLUP results. The highest prediction accuracy from the low LD scenario was 0.30 from RF and 0.36 from GBLUP, and increased to 0.39 for both methods in the high LD population. Random forest successfully identified important SNP in close map distance to QTL explaining a high proportion of the phenotypic trait variations.

Key words: disease trait, random forest methodology, accuracy of genomic prediction

\section{INTRODUCTION}

Classically, large sire calibration groups combined with a 2-step procedure are used to estimate genetic values based on SNP marker effects in dairy and dualpurpose cattle-breeding programs (Edel et al., 2011). Such procedures use highly accurate conventional EBV for sires, implying consideration of the traditional flow of traits from official recording systems. Modern dairy cattle-breeding programs are aiming at including novel functional traits that reflect animal health, behavior, and product quality (König et al., 2013).

From an animal breeding perspective, substantial cow health improvements and increasing monetary genetic gain imply the direct inclusion of health traits into overall breeding goals or selection indices (König et al., 2009; Egger-Danner et al., 2012). However, for novel traits, and without the organized and widespread progeny testing schemes of the past, sires have a limited number of daughter records. Only a few daughter records per sire imply sire EBV with low reliability, as well as inaccurate genomic predictions from 2-step procedures. The reliability of genomic predictions can be improved by including genotyped females in the training set (Pryce et al., 2010; Mc Hugh et al., 2011), 
or by setting up large cow calibration groups based solely on cow phenotypes (Pimentel et al., 2013). In a study by Buch et al. (2012), the accuracy of direct genomic breeding values (GEBV) was higher when using cow phenotypes rather than sire EBV in training sets. Higher numbers of genotyped females allow more accurate selection of bull dams and cow dams, with an associated increase of genetic gain in the whole population (Thomasen et al., 2014).

Cow calibration groups - that is, combining phenotypes for novel traits with high-density genetic markers - offer a new perspective on breeding for improved disease resistance. Still, disease-related traits are generally categorical, influenced by multiple genes, deviate from Mendelian inheritance, and show obvious gene-bygene and gene-by-environment interactions (Hernandez and Blazer, 2006), all of which pose statistical challenges for GEBV estimation. Classically, as introduced in the key paper by Meuwissen et al. (2001), mixed model equations have been solved by applying genomic BLUP (GBLUP) models or Bayesian methodology. But especially for novel traits, current cow calibration group studies are characterized by a small number of genotyped animals $(n)$ genotyped with dense SNP panels $(m)$ (Kramer et al., 2014). This implies model overparameterization ( $m$ larger than $n$ ), suggesting that an alternative methodology should be evaluated, such as random forest (RF) applications.

In animal genetics, RF has been applied to genomewide association studies to identify SNP associated with phenotypes, and to map QTL on the genome (Minozzi et al., 2014). Compared with other genome-wide association study methods for binary traits (e.g., classification and regression trees, logistic regressions), RF performed better for a large sample size combined with a low percentage of missing data (Garcia-Magarinos et al., 2009). Li et al. (2014) and Nguyen et al. (2015) have also explored the potential of RF for genome-wide association studies. In genomic predictions, GonzálezRecio and Forni (2011) showed that RF performed better than Bayesian regressions in detecting resistant and susceptible animals from genetic markers. Ogutu et al. (2011) applied RF to a calibration group of 2,326 genotyped and phenotyped individuals, and correlations between predicted GEBV and true breeding values were in the moderate range (0.39 to 0.54 ).

A variety of factors and parameters [e.g., the heritability and genetic architecture of the trait, the linkage disequilibrium (LD) structure of the population, and the design of the training set] affect the accuracy of genomic predictions. In particular, the size of the training set and the strength of the genetic relationships between training and testing samples contributed to different prediction accuracies from GBLUP and Bayesian applications (Albrecht et al., 2011; Pszczola et al., 2012; de Los Campos et al., 2013). The efficiency of RF for genomic predictions using calibration groups, and how to stratify data sets according to genomic architectures and trait characteristics, has not yet been clarified. However, knowledge in this area is needed, because cow calibration groups, especially for novel health traits, usually represent only a small subpopulation of selected herds. For example, a calibration group of $\sim 20,000$ cows from only $\sim 50$ herds in the eastern part of Germany is the basis for genomic selection for health traits in the German Holstein population (Yin and König, 2016), but the registered German Holstein population includes 1.72 million cows from 18,700 herds (ADR, 2014). In general, higher disease incidences were reported for cows kept in large-scale herds in the eastern part of Germany in comparison to cows from family farms in the western part (Gernand et al., 2012). König et al. (2005) identified genotype by environment interactions for protein yield when stratifying data according to herd location in either eastern or western Germany. In this context, we hypothesize an effect of cow calibration group characteristics (in cows from large-scale contract herds in eastern Germany) when predicting the disease probability of a genotyped female calf or heifer from a different subpopulation.

We applied stochastic simulations to mimic different designs of cow training and testing sets, the basis for ongoing genomic predictions for disease traits. Specifically, the objectives of the present study were (1) to compare the accuracy of genomic predictions using RF and GBLUP for a simulated binary trait by altering the incidence of diseased cows in the training population, heritability, number of QTL, marker density, and the LD structure of the genotyped population; and (2) to compare RF estimates for the effects and locations of the most important SNP with true QTL.

\section{MATERIALS AND METHODS}

\section{Simulation of Populations}

We used QMSim software (Sargolzaei and Schenkel, 2009) to generate phenotypes, genotypes, and true breeding values in a dairy cattle population by applying stochastic simulations. At first, over a time span of 1,000 generations, we created a historical population including 20,000 females and 400 males to achieve the desired LD level. To generate a population with a higher level of LD, we simulated a "bottleneck." For this purpose, we initiated the same simulation process, but after 1,000 generations, we decreased the population 
Table 1. The evaluated scenarios (I to VII) with respect to the number of markers and QTL, the heritability of the trait, and the level of linkage disequilibrium

\begin{tabular}{lccccccc}
\hline & \multicolumn{7}{c}{ Scenario } \\
\cline { 2 - 8 } Variable & I & II & III & IV & V & VI & VII \\
\hline h $^{2}$ & 0.3 & 0.3 & 0.1 & 0.3 & 0.3 & 0.1 & 0.1 \\
No. of QTL & 725 & 290 & 290 & 725 & 290 & 290 & 290 \\
No. of SNP & $10 \mathrm{~K}$ & $10 \mathrm{~K}$ & $10 \mathrm{~K}$ & $50 \mathrm{~K}$ & $50 \mathrm{~K}$ & $50 \mathrm{~K}$ & $50 \mathrm{~K}$ \\
Level of linkage disequilibrium & Low & Low & Low & Low & Low & Low & High \\
\hline
\end{tabular}

size over 80 generations to 500 individuals. Afterward, the population size increased over 140 generations, resulting again in 20,000 females and 400 males. In the second simulation step, in the recent population, all animals from the last generation of the historical population served as founders. We expanded the recent population by simulating an additional 8 generations using a random mating design. Each mating produced 1 offspring with an equal probability of being either male or female. Replacement rates were $50 \%$ for males and $20 \%$ for females, and selection for both sexes was based on EBV.

In the recent population, 7 different scenarios (I to VII; Table 1) were simulated to reflect variations with regard to heritability, number of QTL, number of SNP, and LD. Biallelic SNP markers were evenly spaced along 29 chromosomes, each $80 \mathrm{cM}$ long. Simulations of either 345 or 1,725 biallelic markers per chromosome depicted applications with 10,005 SNP (10K chip) and 50,025 SNP (50K chip), respectively. For both marker densities $10 \mathrm{~K}$ and $50 \mathrm{~K}, 2$ different numbers of QTL (either 10 or 25 QTL on each chromosome) affected the trait of interest. The QTL effects were sampled from a gamma distribution with a shape parameter of 0.4. The gamma distribution assumes many QTL with small effects and, correspondingly, only a few QTL with large effects. The mutation rate was $2.5 \times 10^{-5}$ for both markers and QTL per locus and per generation, as used in previous simulations (Yin et al., 2014). The whole amount of additive-genetic variance was attributed to the QTL, implying no further polygenic effects. Simulations considered a low $\left(h^{2}=0.10\right)$ and a moderate heritability trait $\left(\mathrm{h}^{2}=0.30\right)$. The whole set of parameters as used for the simulations is summarized in Table 2. Cows of the last generation were ranked in descending order according to the continuous trait. The value from rank number 4,000 was defined as threshold, implying 4,000 animals with code $1=$ diseased, and 16,000 animals with code $0=$ healthy. Such ranking and transformation into a binary outcome reflected a

Table 2. Parameters of the simulation process

\begin{tabular}{|c|c|c|}
\hline Parameter & $\begin{array}{c}\text { Low linkage } \\
\text { disequilibrium }\end{array}$ & $\begin{array}{c}\text { High linkage } \\
\text { disequilibrium }\end{array}$ \\
\hline \multicolumn{3}{|l|}{ Historical population } \\
\hline No. of generations (population size) in phase 1 & $1,000(20,400)$ & $1,000(20,400)$ \\
\hline No. of generations (population size) in phase 2 & - & $1,080(500)$ \\
\hline No. of generation (population size) in phase 3 & - & $1,220(20,400)$ \\
\hline \multicolumn{3}{|l|}{ Recent population } \\
\hline No. of founder sires (dams) & \multicolumn{2}{|c|}{$400(20,000)$} \\
\hline No. of generations & \multicolumn{2}{|c|}{8} \\
\hline No. of offspring per dam & \multirow{2}{*}{\multicolumn{2}{|c|}{$\begin{array}{c}1 \\
\text { Random }\end{array}$}} \\
\hline Mating system & & \\
\hline Replacement ratio for males (females) & \multicolumn{2}{|c|}{$0.5(0.2)$} \\
\hline Criteria for selection/culling & \multicolumn{2}{|c|}{$\mathrm{EBV} /$ age } \\
\hline Sex probability for offspring & \multicolumn{2}{|c|}{0.5} \\
\hline \multicolumn{3}{|l|}{ Genome } \\
\hline No. of chromosomes & \multicolumn{2}{|c|}{29} \\
\hline Total length of chromosomes (cM) & \multicolumn{2}{|c|}{2,320} \\
\hline Marker distribution & \multicolumn{2}{|c|}{ Evenly spaced } \\
\hline No. of QTL alleles & \multicolumn{2}{|c|}{ Random $(2,3$, or 4$)$} \\
\hline Effects of QTL alleles & \multirow{2}{*}{\multicolumn{2}{|c|}{ Gamma $(0.4)$}} \\
\hline Marker and QTL mutation rate & & \\
\hline Position of marker and QTL & \multicolumn{2}{|c|}{ Random } \\
\hline No. of QTL & 290,725 & 290 \\
\hline No. of markers & $10,005,50,025$ & 50,025 \\
\hline Heritability of the trait & $0.30,0.10$ & 0.10 \\
\hline
\end{tabular}


selection strategy on a continuous trait (e.g., protein yield) in the past generations, and a pronounced antagonistic relationship with a disease trait.

The LD measurement applied in this study was $\mathrm{r}^{2}$ (squared correlation coefficient according to Hill and Robertson, 1968). Average $r^{2}$ in the low-LD scenario (VI) was 0.224 for distances of $0.05 \mathrm{cM}$, and the corresponding average $\mathrm{r}^{2}$ in the high-LD scenario (VII) was 0.425 . We used the PLINK program (Purcell et al., 2007) to calculate the amount of LD between the most important QTL (10 QTL on the whole genome explaining the highest amount of genetic variance in relation to the phenotypic variance of the trait), and the most important SNP at the same chromosome identified by applying RF methodology.

\section{Prediction of GEBV and of SNP Effects}

Genomic Breeding Values. To estimate GEBV, we applied GBLUP and RF to the simulated binary data. For GBLUP, we used the AI-REML algorithm (DMU software package, Madsen and Jensen, 2010), which allows the specification of a generalized linear mixed model with a logit link function for binary data. The statistical model was

$$
\begin{aligned}
\operatorname{logit}\left(\pi_{r}\right) & =\log \left[\pi_{r} /\left(1-\pi_{r}\right)\right] \\
& =\phi+\gamma_{r},
\end{aligned}
$$

where $\pi_{r}=$ probability of occurrence for the disease of cow $r, \phi=$ overall mean effect, and $\gamma_{r}=$ random animal effect.

Random animal effect was included by considering the genomic relationships among animals based on SNP marker data. The genomic relationship matrix (G matrix) was constructed according to the method proposed by VanRaden (2008) and applying the $\mathbf{G}$ matrix software (Su and Madsen, 2013). Markers with minor allele frequency $<0.05$ were discarded. A small value (0.01) was added to the diagonal of the $\mathbf{G}$ matrix to circumvent problems with matrix singularity.

For RF analyses, we applied the java package RanFoG (González-Recio and Forni, 2011). In the RF analysis (Breiman, 2001), thousands of classification trees were constructed by bootstrapping (Efron and Tibshirani, 1993) of the data in the training set. For the construction of each tree, RF used on average about two-thirds of the observations and a random subset $p$ of the $m$ SNP $(p \sim 2 / 3 \times m)$. Cows not included in the bootstrapped sample were defined as "out of bag" (OOB), being the testing set for each tree. At each node, data were split in 2 branches based on the genotype at $\operatorname{SNP} j$ by minimizing a loss function for classification. Repetition of this procedure implied a large number of trees (i.e., RF), until the convergence criterion was achieved. The convergence criterion used classification errors of OOB samples. In the present study, 2,000 and 5,000 trees were constructed for the $10 \mathrm{~K}$ and the $50 \mathrm{~K}$ SNP chips, respectively. Random sampling (bootstrapping) of the data contributed to the formation of decorrelated trees. Each tree reflected the most frequent outcome of the disease for a given combination of SNP genotypes. The average of the predicted value of each tree was the probability of being susceptible to the disease.

Assessing Prediction Performance. When allocating cows to training and testing sets, we considered 20,000 cows from the last 2 generations. All cows had genotypes, but phenotypes in the testing set were assumed unknown. The training and testing sets were designed to include different percentages of sick and healthy cows (Figure 1). In total, 4,000 cows were sick, and 16,000 cows were healthy, reflecting a population disease incidence of 0.20 . Initially, the 4,000 sick cows were allocated to the testing set, and all 16,000 cows in the training set were healthy. In ongoing animal allocation schemes, the number of sick cows in the training set increased by moving 400 sick animals from the testing to the training set. To keep the size of both sets constant, 400 healthy cows were then moved from the training to the testing set. This was done stepwise in increments of 400 cows, until all of the 4,000 sick cows had been moved to the training set. By the final step, all 4,000 cows in the testing set were healthy. For all allocation schemes in each scenario, we performed 10 replicates.

The correlation coefficient between true breeding values and predicted GEBV from cows in the testing set was the evaluation criterion for the prediction accuracy of GEBV. A further evaluation criterion for phenotype predictions was the area under the receiving operating characteristic curve (AUROC); AUROC is an information criterion for assessing the efficiency of classification models by comparing true positive and false positive classifications, and it has often been used to compare models in clinical studies. As specified by González-Recio et al. (2014), the genetic susceptibility to disease was predicted based on SNP genotypes. Accordingly, animals were classified as either susceptible or nonsusceptible, depending on an arbitrary GEBV threshold (t). Genomic predictions in relation to the true genetic susceptibility could either be true positive, false positive, true negative, or false negative, classified as positive $[\mathbf{F P R}=$ (predicted positives) over (negatives); $\mathbf{T P R}=$ (predicted positives) over (positives)] The receiver operating characteristic curve depicted 


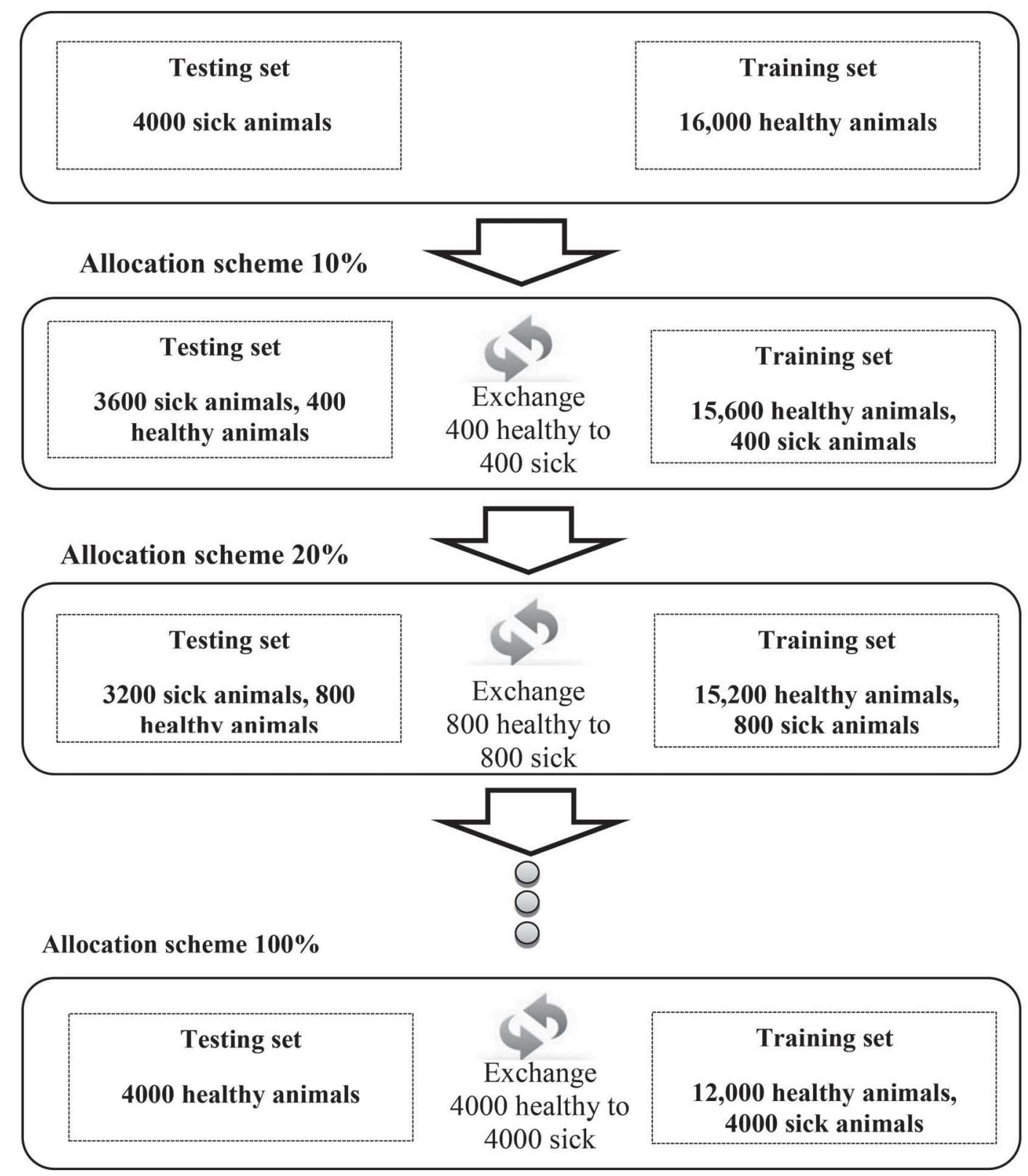

Figure 1. Strategy for the creation of training and testing sets.

TPR in relation to FPR for all thresholds in $(0,1)$ that could be used to classify animals as sick and healthy.

Importance of Single SNP. The importance of single SNP was calculated based on OOB data, comparing the difference in prediction accuracy from the original OOB sample of a given tree in relation to another OOB sample where the genotype of the $j$ th SNP was permuted. The average decrease in accuracy for the $j$ th permuted SNP over all bootstrapped trees determined the importance of each SNP. The relative importance of a single SNP in the range from 0 to 1 was calculated by defining a SNP ratio, which was a specific single SNP value in relation to the highest SNP value (most important SNP: value of 1). Values of all SNP were saved in the variable importance file of RanFoG.

\section{RESULTS AND DISCUSSION}

\section{Accuracy of Predictions with Lower (10K) and Higher Density (50K) SNP Chips}

General Aspects. For the 10K SNP panel, accuracies of cow GEBV in the testing set estimated with RF and GBLUP are listed in Table 3 (scenarios I, II, and 
Table 3. Correlation between true and predicted genomic breeding values for scenarios I (10K SNP, $\mathrm{h}^{2}=0.30$ and 725 QTL), II (10K SNP, $\mathrm{h}^{2}$ $=0.30$ and $290 \mathrm{QTL})$, and III (10K SNP, $\mathrm{h}^{2}=0.10$ and $\left.290 \mathrm{QTL}\right)$ from random forest (RF) and genomic BLUP (GBLUP) applications (values in parentheses show the SD from 10 replicates)

\begin{tabular}{|c|c|c|c|c|c|c|c|c|c|c|c|}
\hline Method & Scenario & \multicolumn{10}{|c|}{ Percentage of sick animals allocated to the training set/percentage of sick animals within the training set ${ }^{1}$} \\
\hline \multirow[t]{3}{*}{$\overline{\text { GBLUP }}$} & I & $\begin{array}{c}0.25 \\
(0.05)\end{array}$ & $\begin{array}{c}0.31 \\
(0.06)\end{array}$ & $\begin{array}{c}0.36 \\
(0.04)\end{array}$ & $\begin{array}{c}0.42 \\
(0.03)\end{array}$ & $\begin{array}{c}0.45 \\
(0.03)\end{array}$ & $\begin{array}{c}0.47 \\
(0.02)\end{array}$ & $\begin{array}{c}0.46 \\
(0.03)\end{array}$ & $\begin{array}{c}0.47 \\
(0.03)\end{array}$ & $\begin{array}{c}0.47 \\
(0.02)\end{array}$ & $\begin{array}{c}0.46 \\
(0.02)\end{array}$ \\
\hline & II & $\begin{array}{c}0.24 \\
(0.06)\end{array}$ & $\begin{array}{c}0.33 \\
(0.04)\end{array}$ & $\begin{array}{c}0.35 \\
(0.001)\end{array}$ & $\begin{array}{c}0.42 \\
(0.03)\end{array}$ & $\begin{array}{c}0.45 \\
(0.02)\end{array}$ & $\begin{array}{c}0.46 \\
(0.03)\end{array}$ & $\begin{array}{c}0.47 \\
(0.03)\end{array}$ & 0.48 & 0.48 & 0.46 \\
\hline & III & 0.16 & 0.24 & $\begin{array}{l}(0.001) \\
0.26\end{array}$ & $\begin{array}{c}(0.03) \\
0.31\end{array}$ & $\begin{array}{c}(0.02) \\
0.31\end{array}$ & $\begin{array}{c}(0.03) \\
0.32\end{array}$ & $\begin{array}{c}(0.03) \\
0.33\end{array}$ & $\begin{array}{c}(0.03) \\
0.35\end{array}$ & $\begin{array}{c}(0.03) \\
0.34\end{array}$ & $\begin{array}{c}(0.02) \\
0.33\end{array}$ \\
\hline \multirow{5}{*}{$\mathrm{RF}$} & & $(0.01)$ & $(0.01)$ & $(0.03)$ & $(0.02)$ & $(0.02)$ & $(0.03)$ & $(0.02)$ & $(0.01)$ & $(0.02)$ & $(0.01)$ \\
\hline & II & 0.13 & 0.20 & 0.25 & 0.29 & 0.29 & 0.32 & 0.34 & 0.35 & 0.34 & 0.32 \\
\hline & & $(0.02)$ & $(0.03)$ & $(0.04)$ & $(0.02)$ & $(0.02)$ & $(0.03)$ & $(0.03)$ & $(0.03)$ & $(0.02)$ & $(0.02)$ \\
\hline & III & 0.07 & 0.11 & 0.14 & 0.16 & 0.19 & 0.21 & 0.20 & 0.23 & 0.23 & 0.23 \\
\hline & & $(0.02)$ & $(0.01)$ & $(0.03)$ & $(0.02)$ & $(0.01)$ & $(0.02)$ & $(0.01)$ & $(0.01)$ & $(0.03)$ & $(0.01)$ \\
\hline
\end{tabular}

${ }^{1}$ Sizes of training and testing sets were 16,000 and 4,000 , respectively, for all scenarios.

III). For all percentages of sick cows in the training set, prediction accuracies from GBLUP always outperformed those from corresponding RF applications. As indicated by smaller $\mathrm{SD}$, results from $\mathrm{RF}$ across the 10 replicates were more homogeneous compared with GBLUP applications. When increasing the number of sick cows allocated to training sets from $10 \%$ (400 cows, or $2.5 \%$ of all cows in the training set are sick) to $60 \%$ $(2,400$ cows, or $15 \%$ of all cows in the training set are sick), the prediction accuracy increased correspondingly. For instance, in scenario I, the prediction accuracy increased from 0.25 to 0.47 for GBLUP, and from 0.14 to 0.32 for RF. The highest accuracies were observed for percentages of sick cows in training sets that were close to the population disease incidence (20\%). The prediction accuracy remained constant when allocating a higher number of sick cows to the training set. The AUROC values for scenarios I, II, and III are summa- rized in Table 4. As well, the AUROC evaluation criterion reflected the superiority of GBLUP compared with RF. The AUROC from GBLUP ranged between 0.56 and 0.66, and was slightly lower for RF. The AUROC value increased only slightly when a higher number of sick animals were allocated to training sets.

For 10K SNP chip panels and in comparison to RF, GBLUP provided more accurate GEBV and differentiated more accurately between sick and healthy individuals. González-Recio and Forni (2011) simulated a moderate heritability trait $\left(\mathrm{h}^{2}=0.25\right)$. They randomly selected 2,500 animals to establish a training set and evaluate the efficiency of Bayesian regressions and machine-learning algorithms for a binary trait. They reported an accuracy of 0.36 when applying RF, and accuracies ranging from 0.37 to 0.41 for boosting algorithms. Ogutu et al. (2011) applied 3 machine-learning methods and ridge-regression BLUP to a calibration

Table 4. The area under the receiving operating characteristic curve (AUROC) for scenarios I (10K SNP, $\mathrm{h}^{2}=0.30$ and $\left.725 \mathrm{QTL}\right)$, II (10K SNP, $\mathrm{h}^{2}=0.30$ and $\left.290 \mathrm{QTL}\right)$, and III (10K SNP, $\mathrm{h}^{2}=0.10$ and 290 QTL) from random forest (RF) and genomic BLUP (GBLUP) applications (the values in parentheses show the SD from 10 replicates)

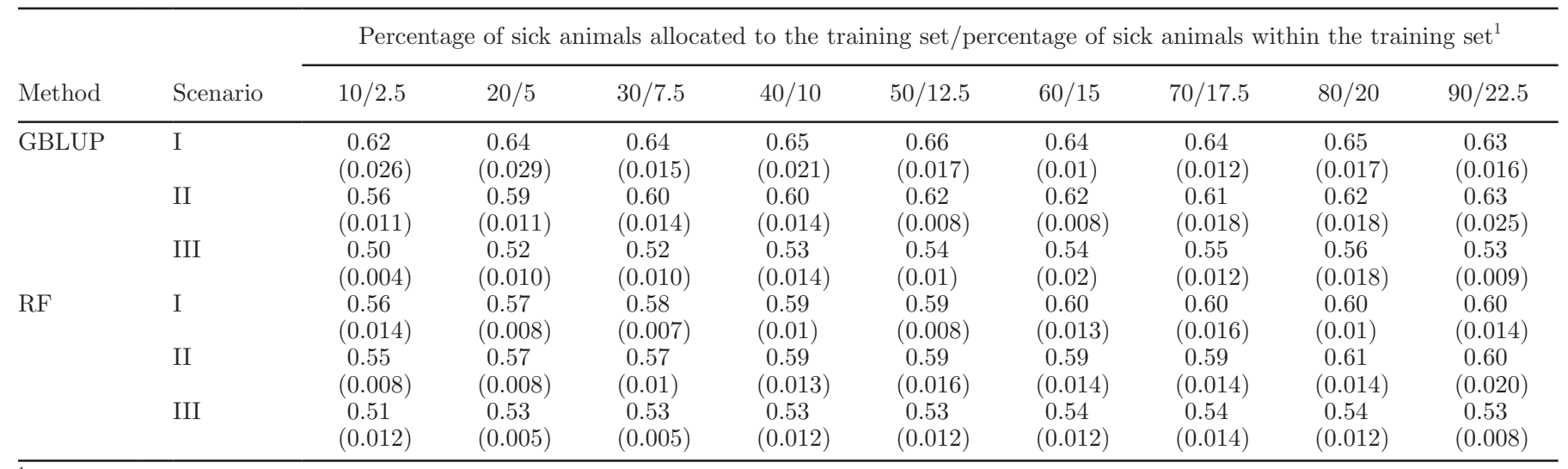

\footnotetext{
${ }^{1}$ Size of training and testing sets were 16,000 and 4,000, respectively, for all scenarios.
} 
group of 2,326 animals genotyped for 10K. For a simulated quantitative trait with heritabilities of 0.39 for females and 0.52 for males, the correlation between predicted GEBV and true breeding values from RF applications was 0.48 , and was 0.60 for ridge regression.

For the 50K SNP chip and for both RF and GBLUP, correlations between GEBV and true breeding values for individuals without phenotypes from scenarios IV, V, VI and VII are listed in Table 5. For all scenarios, prediction accuracies increased with the percentage of sick animals allocated to the training set until a $50 \%$ threshold was achieved, and remained stable thereafter. For scenario IV and GBLUP applications, even a continuous increase was observed, with highest accuracies for the $100 \%$ sick animal allocation scheme. Specifically, for GBLUP and scenario IV, prediction accuracies increased from 0.24 (10\% of sick animals allocated to the training set) to 0.50 (100\% of sick animals allocated to the training set, or $25 \%$ of sick animals within the training set). With scenario IV and RF methodology, accuracies increased from 0.20 to 0.52 , and the highest accuracy (0.53) was identified for 3,200 sick animals allocated to the training set ( $80 \%$ of sick cows allocated to the training set). Such a high percentage of sick animals allocated to the training set reflected similar disease incidences for the training set and the whole population. Also, AUROC evaluation supported the better performance of RF compared with GBLUP for scenarios with the 50K SNP chip (Table 6). For both RF and GLUP, AUROC was lowest for low percentages of sick animals allocated to the training set. For example, in scenario IV, and for only $10 \%$ of sick animals allocated to training sets, AUROC values were 0.58 and 0.57 when using RF and GBLUP, respectively.

With regard to the 50K SNP chip and scenario IV, $\mathrm{RF}$ performed better than GBLUP for all allocation schemes, apart from the 10, 30, and $90 \%$ of cases. Increased marker density contributed to RF prediction improvements. An increase of SNP markers shortened the distances between markers and functional mutations. In consequence, SNP close to a QTL were sampled with sufficient frequency, implying that the signal of the QTL is captured by distinct SNP in close map distance. The higher prediction accuracies with larger marker density were in agreement with previous studies (Makowsky et al., 2011; Vazquez et al., 2012). However, VanRaden et al. (2011) identified only minor increases in prediction accuracies when using SNP chips larger than $50 \mathrm{~K}$.

When using the 50K SNP to analyze binary data, $\mathrm{RF}$ ranked individuals more accurately than GBLUP, and also distinguished precisely between healthy and affected individuals in most allocating schemes. For example, AUROC 0.66 (scenario IV, method RF, $80 \%$ of sick animals allocated to the training set) indicated for $66 \%$ of susceptible individuals larger genomic predictions than for nonsusceptible individuals. Likewise, an AUROC of value 0.50 implied that a classification in either being sick or healthy was no better than a random guess: $50 \%$ of susceptible individuals with larger predictions than non-susceptible individuals, and vice versa. AUROC values were in the range of previous studies (González-Recio and Forni, 2011; Vazquez et al., 2012), which reported AUROC between 0.58 and

Table 5. Correlation between true and predicted genomic breeding values for scenarios IV (50K SNP, $\mathrm{h}^{2}=0.30$ and $\left.725 \mathrm{QTL}\right), \mathrm{V}\left(50 \mathrm{~K}\right.$ SNP, $\mathrm{h}^{2}$ $=0.30$ and $290 \mathrm{QTL})$, VI (50K SNP, $\mathrm{h}^{2}=0.10$ and $\left.290 \mathrm{QTL}\right)$, and VII (50K SNP, $\mathrm{h}^{2}=0.10,290$ QTL and high linkage disequilibrium) from random forest (RF) and genomic BLUP (GBLUP) applications (the values in parentheses show the SD from 10 replicates)

Percentage of sick animals allocated to the training set/percentage of sick animals within the training set ${ }^{1}$

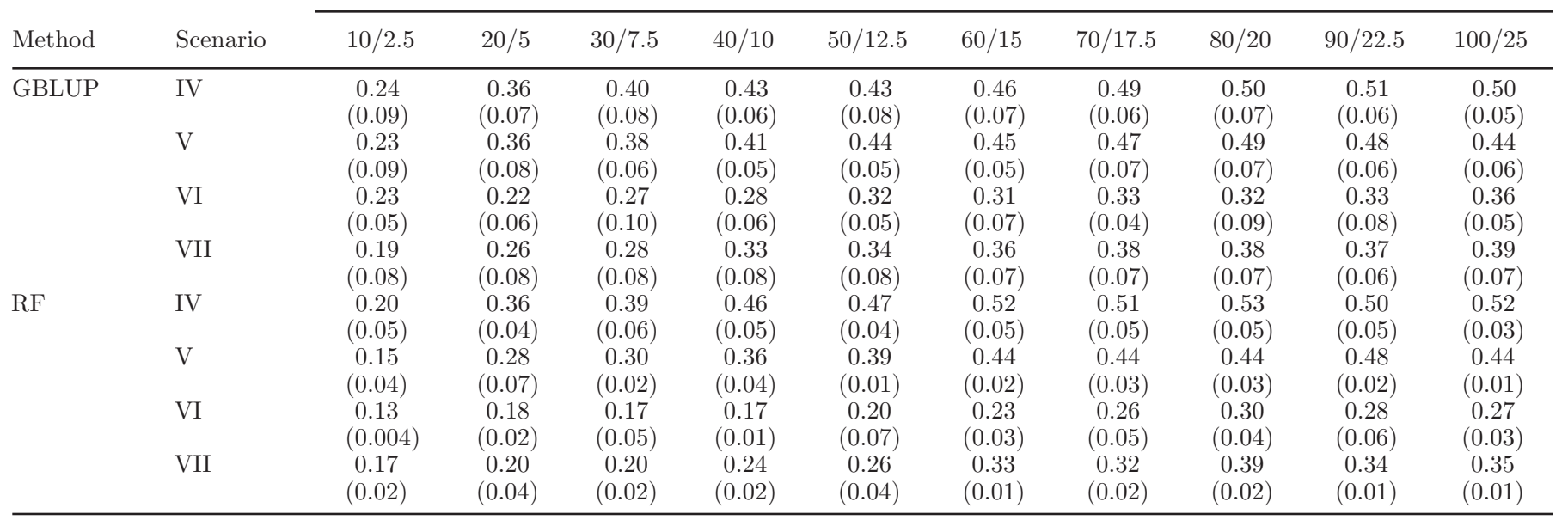

${ }^{1}$ Size of training and testing sets were 16,000 and 4,000, respectively, for all scenarios. 
0.70. Nguyen et al. (2015) calculated larger AUROC, in the range of 0.62 to 0.97 , when using high-density 400K SNP chips. Hence, marker density and the size of training sets were identified as the most important parameters effecting AUROC.

Interestingly, and presumably attributed to the large-scale cow calibration group, accuracies of GEBV in the present study were higher than previous simulation evaluations. In contrast, values for AUROC were slightly smaller. Using RF, prediction was based on a random subsample of SNP. Therefore, especially for low-density SNP panels, it might be that SNP close to a QTL were not sufficiently sampled, implying that the QTL signal was captured by a distant SNP.

We observed a wide range of computation times across scenarios. Computation time was generally higher for RF. For example for scenario I, the computation time for GBLUP was $46 \mathrm{~h}$ per replicate, but $68 \mathrm{~h}$ per replicate for RF. Ghafouri-Kesbi et al. (2016) reported that GBLUP was faster than RF, and Neves et al. (2012) confirmed the lower computation time for a GBLUP model compared with RF. Computation time might be the crucial limiting factor when intending to apply RF to a large data set of genotyped cows.

Effect of the Number of QTL. For the 10K SNP panel, accuracies of GEBV from RF and GBLUP applications for scenarios with identical heritability but differing numbers of QTL [i.e., scenario I (725 QTL) vs. II (290 QTL)] were very similar (Table 3). For the low-density 10K SNP panel, with either a low or larger number of QTL (scenario I vs. II), prediction accuracies from GBLUP were higher than from RF. We also identified a minor effect on AUROC when decreasing the number of QTL (Table 4). The effect of QTL reduction on AUROC was more pronounced for GBLUP, but AUROC still indicated the superiority of GBLUP over $\mathrm{RF}$ for a reduced number of QTL.

For the 50K SNP panel, the accuracies of GEBV from RF and GBLUP applications for scenarios IV (725 QTL) and V (290 QTL), both considering a trait with heritability 0.30 , are shown in Table 5 . Especially for $\mathrm{RF}$, we identified a pronounced effect of the QTL number on prediction accuracies: they strongly decreased for all animal allocation schemes when decreasing the number of QTL from 725 to 290. Regarding GBLUP, we identified only a slight reduction in prediction accuracies. For most of the cow allocation schemes (apart from the $10 \%, 30 \%$, and $90 \%$ cases) and 725 simulated QTL, RF performed better than GBLUP, but we had the opposite result when simulating 290 QTL. Calculated AUROC values for scenarios IV and V are summarized in Table 6. An evaluation of AUROC exhibited better RF performance for both QTL scenarios. Also for the lower number of 290 QTL and despite lower correlations between true breeding values and GEBV, the RF method separated healthy and sick individuals more precisely than GBLUP (comparison of results for scenario $\mathrm{V}$ in Tables 6 and 7).

For a large number of QTL, Ghafouri-Kesbi et al. (2016) reported higher GBLUP accuracies compared with accuracies from RF and gradient boosting. For their scenario with a low number of QTL and allele effects drawn from a gamma distribution, gradient boosting was the best-performing method. For all methods and in contrast to the results from the present study, Ghafouri-Kesbi et al. (2016) reported generally increas-

Table 6. The area under the receiving operating characteristic curve (AUROC) for scenarios IV (50K SNP, $\mathrm{h}^{2}=0.30$, and $725 \mathrm{QTL}$ ), V (50K SNP, $\mathrm{h}^{2}=0.30$, and 290 QTL), VI (50K SNP, $\mathrm{h}^{2}=0.10$, and 290 QTL), and VII (50K SNP, $\mathrm{h}^{2}=0.10,290$ QTL, and high linkage disequilibrium) from random forest (RF) and genomic BLUP (GBLUP) applications (values in parentheses show the SD from 10 replicates)

Percentage of sick animals allocated to the training set/percentage of sick animals within the training set ${ }^{1}$

\begin{tabular}{|c|c|c|c|c|c|c|c|c|c|c|}
\hline Method & Scenario & $10 / 2.5$ & $20 / 5$ & $30 / 7.5$ & $40 / 10$ & $50 / 12.5$ & $60 / 15$ & $70 / 17.5$ & $80 / 20$ & $90 / 22.5$ \\
\hline \multirow[t]{7}{*}{ GBLUP } & IV & $\begin{array}{c}0.57 \\
(0.06)\end{array}$ & $\begin{array}{c}0.59 \\
(0.036)\end{array}$ & $\begin{array}{l}0.60 \\
(0.041)\end{array}$ & $\begin{array}{l}0.59 \\
(0.031)\end{array}$ & $\begin{array}{l}0.60 \\
(0.037)\end{array}$ & $\begin{array}{c}0.61 \\
(0.042)\end{array}$ & $\begin{array}{l}0.63 \\
(0.031)\end{array}$ & $\begin{array}{c}0.63 \\
(0.040)\end{array}$ & $\begin{array}{c}0.64 \\
(0.046)\end{array}$ \\
\hline & $\mathrm{V}$ & 0.53 & 0.58 & 0.59 & 0.60 & 0.60 & 0.60 & 0.62 & 0.61 & 0.62 \\
\hline & & $(0.06)$ & $(0.05)$ & $(0.05)$ & $(0.02)$ & $(0.02)$ & $(0.02)$ & $(0.02)$ & $(0.04)$ & $(0.03)$ \\
\hline & VI & 0.54 & 0.52 & 0.53 & 0.53 & 0.53 & 0.53 & 0.54 & 0.55 & 0.53 \\
\hline & & $(0.04)$ & $(0.05)$ & $(0.03)$ & $(0.02)$ & $(0.02)$ & $(0.02)$ & $(0.02)$ & $(0.04)$ & $(0.04)$ \\
\hline & VII & 0.56 & 0.56 & 0.57 & 0.57 & 0.57 & 0.58 & 0.58 & 0.58 & 0.57 \\
\hline & & $(0.019)$ & $(0.020)$ & $(0.023)$ & $(0.025)$ & $(0.010)$ & $(0.019)$ & $(0.019)$ & $(0.014)$ & $(0.007)$ \\
\hline \multirow[t]{8}{*}{$\mathrm{RF}$} & IV & 0.58 & 0.64 & 0.64 & 0.66 & 0.66 & 0.63 & 0.64 & 0.66 & 0.64 \\
\hline & & $(0.01)$ & $(0.02)$ & $(0.02)$ & $(0.02)$ & $(0.035)$ & $(0.041)$ & $(0.021)$ & $(0.012)$ & $(0.009)$ \\
\hline & $\mathrm{V}$ & 0.47 & 0.58 & 0.57 & 0.61 & 0.62 & 0.62 & 0.62 & 0.63 & 0.65 \\
\hline & & $(0.02)$ & $(0.02)$ & $(0.01)$ & $(0.01)$ & $(0.02)$ & $(0.02)$ & $(0.01)$ & $(0.02)$ & $(0.03)$ \\
\hline & VI & 0.57 & 0.54 & 0.54 & 0.56 & 0.56 & 0.56 & 0.57 & 0.57 & 0.59 \\
\hline & & $(0.05)$ & $(0.01)$ & $(0.01)$ & $(0.02)$ & $(0.01)$ & $(0.01)$ & $(0.01)$ & $(0.01)$ & $(0.004)$ \\
\hline & VII & 0.56 & 0.55 & 0.56 & 0.56 & 0.56 & 0.59 & 0.57 & 0.58 & 0.58 \\
\hline & & $(0.026)$ & $(0.016)$ & $(0.026)$ & $(0.018)$ & $(0.011)$ & $(0.011)$ & $(0.018)$ & $(0.024)$ & $(0.011)$ \\
\hline
\end{tabular}

\footnotetext{
${ }^{1}$ Size of training and testing sets were 16,000 and 4,000, respectively, for all scenarios.
} 
ing prediction accuracies with a decreasing number of QTL. Reasons for these opposing results might be the different number of simulated chromosomes, and different effective population sizes. Ghafouri-Kesbi et al. (2016) spaced 10,000 SNP along 5 chromosomes and considered an effective population size $\left(N_{e}\right)$ of 100. According to Daetwyler et al. (2010a), prediction accuracy depends on the number of independent chromosome segments $\left(\boldsymbol{M}_{e}\right)$, and $M_{e}$ itself depends on the effective population size and the length of the genome (Goddard, 2009). González-Recio and Forni (2011) identified higher accuracies for GEBV with Bayesian regression applications than with boosting algorithms and RF methods when the number of QTL increased. In the present study, RF showed the smallest accuracy but the highest AUROC for a scenario, assuming a large number of QTL. In this context, González-Recio and Forni (2011) discussed and compared both evaluation criteria AUROC and the accuracy of GEBV. Irrespective of lower accuracies when applying RF to a trait characterized by a high number of QTL, RF technique was more accurate for discerning between healthy and affected individuals. Generally, the higher sensitivity of RF on QTL alterations than GBLUP (quite stable results for both QTL scenarios) can be explained as follows: GBLUP assumed the same variance for each independent chromosome segment regardless of the effect of the segment, and RF based on a sampling technique for predictors (SNP). Therefore, by using high-density marker panels combined with a large number of QTL, SNP in close distance to a QTL were sufficiently frequently sampled. Nevertheless, most of the important dairy traits are affected by many genes with small effects, and this supports the assumptions made for GBLUP applications (Hayes et al., 2009). GBLUP results from the present study are in agreement with Daetwyler et al. (2010b), who reported independence of prediction accuracies on the number of QTL.

Effect of Heritability. Studying the effect of heritability on prediction accuracies and on AUROC for the 10K SNP chip, and the same number of 290 QTL, implies a comparison of II with III (prediction accuracy in Table 3, AUROC in Table 4). A heritability decrease was associated with a pronounced decrease in prediction accuracy for all allocation schemes, and for both GBLUP and RF. For GBLUP and RF, and for $h^{2}=$ 0.10 , the highest prediction accuracies were observed for an extremely high percentage of sick animals allocated to the training set $(80,90$, or $100 \%)$. For $\mathrm{h}^{2}=$ 0.30 , high accuracies were also identified for intermediate percentages of sick animals allocated to training sets (i.e., for the 50 or $60 \%$ threshold). We identified an obvious decrease for AUROC when lowering the heritability from 0.30 to 0.10 (Table 4). This decrease was more pronounced for GBLUP than for RF. Similar AUROC were identified from both methods when $\mathrm{h}^{2}=$ 0.10 and when allocating more than $40 \%$ of sick animals to training sets. Hence, both methods GBLUP and RF revealed the same potential to distinguish between sick and healthy animals for low heritability binary traits according to AUROC, but prediction accuracies were greater for GBLUP than for RF.

The effect of heritabilities $\left(\mathrm{h}^{2}=0.30\right.$ and $\left.\mathrm{h}^{2}=0.10\right)$ on accuracies of GEBV is depicted in Table 5 for 290 QTL and 50,025 SNP (comparison of scenarios V and VI). As expected, heritability decrease was associated with decreased accuracies for both GBLUP and RF, and for all cow allocation schemes. Accuracy decrease was more obvious for RF than for GBLUP. Calculated AUROC for both GBLUP and RF, within both scenarios V and VI, are given in Table 6. Also here, apart from an extremely low percentage of sick animals in the training set (10\% allocation scheme), the comparison of scenarios V and VI displayed higher AUROC for the higher heritability scenario V. In the low heritability scenario VI, AUROC values from RF were higher than from GBLUP applications. For a moderate heritability of 0.30 (scenario V), GBLUP partially performed better, especially for low percentages of sick animals in the training set.

The detrimental effect of decreasing heritabilities on GEBV accuracies using GBLUP has been proved in several previous studies (Daetwyler et al., 2010b; Zhang et al., 2010). Daetwyler et al. (2013) found an increasing effect of heritabilities on prediction accuracies with an increasing number of QTL, and they deterministi-

Table 7. The calculated $\mathrm{r}^{2}$ (squared correlation coefficient) between each of 10 most effective QTL and the most important SNP located in the same chromosome for scenarios I $\left(10 \mathrm{~K}\right.$ SNP chip, $\mathrm{h}^{2}=0.3$, and QTL $\left.=725\right)$ and IV (50K SNP chip, $\mathrm{h}^{2}=0.3$, and QTL $\left.=725\right)$

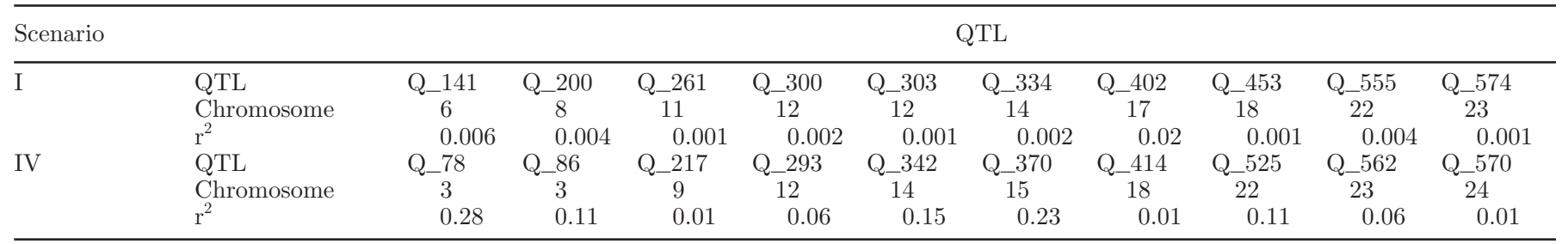


cally assessed the prediction accuracy: $\sqrt{N_{p} h^{2} /\left(N_{p} h^{2}+M_{e}\right)}$, where $N_{p}$ was the number of animals in the training. Guo et al. (2014) identified associations between prediction accuracies and genomic heritabilities of training and testing sets. The increasing genomic heritability was due to higher genetic variations in training and testing sets, contributing to accurate predictions of marker effects.

Effect of LD Structure. Scenario VII was simulated to evaluate the effect of $\mathrm{LD}$ on accuracies of GEBV and on AUROC. Remaining parameters (i.e., a heritability of 0.10 and 290 QTL) were identical to scenario VI. Average $\mathrm{r}^{2}$ in the low LD scenario (VI) was 0.224 at distances of $0.05 \mathrm{cM}$, and the corresponding average $r^{2}$ in the high LD scenario (VII) was 0.425 . With higher LD, accuracies of GEBV increased for all animal allocation schemes and for both GBLUP and RF (Table 5). Only in the extreme $10 \%$ allocation scheme and GBLUP, prediction accuracy was slightly higher for the low LD population (0.23 vs. 0.19$)$. Generally, gain in accuracies for the high LD scenario was more pronounced for RF than for GBLUP. With regard to VII, highest accuracies were identified for 80 and $100 \%$ of sick animals allocated to the training set when applying RF and GBLUP, respectively. A higher level of LD between SNP and QTL implies that more markers capture a higher proportion of the genetic variance (Goddard, 2009), a prerequisite for an efficient performance of RF. Results from GBLUP were in agreement with simulations by Yin et al. (2014), who also reported increasing accuracies of GEBV with increasing LD, and with increasing marker density. Meng et al. (2009) simulated SNP with different effects on a given disease. They identified a strong effect of LD on RF performance when simulating SNP with large genetic effects. Daetwyler et al. (2010b) also studied the effect of the genomic architecture of traits and reported a strong LD influence on the accuracies of GEBV. Results from the present study revealed a stronger effect of LD on prediction accuracies when applying RF than when applying GBLUP.

\section{Association Mapping}

Results from predictive models (e.g., from genomic predictions) can be used for association mapping (e.g., Biffani et al., 2015), in general, and this is true specifically for RF applications. The relative importance of the 10,005 SNP generated in scenario I by RF is shown in Figure 2a for 1 specific replicate. Figure 2a depicts the 10 QTL that explain the highest amount of genetic variance in relation to the phenotypic variance of the trait. Generally, small distances between the most im- portant SNP with 1 of the 10 top QTL were identified. For example, the second effective QTL was located on chromosome 6 at $19.64 \mathrm{cM}$, and the most important SNP identified by RF was located at $22.67 \mathrm{cM}$ at the same chromosome. The short distance between the most effective QTL and the most important SNP indicates that RF can identify major QTL with high accuracy. The efficiency of RF in identifying the most effective QTL was further improved by increasing the number of $\mathrm{SNP}$ from $10 \mathrm{~K}$ to $50 \mathrm{~K}$ in scenario IV (Figure $2 \mathrm{~b}$ ). The physical distances between the most important SNP identified by RF and the 10 most effective QTL decreased, and the 4 important QTL directly overlapped with the 10 important SNP. For example, one of the most important QTL was located on chromosome 3 at $61.84 \mathrm{cM}$, and the most important SNP identified by $\mathrm{RF}$ on the same chromosome was located at $63.47 \mathrm{cM}$.

Levels of LD between 10 most important SNP identified using RF in scenarios I and IV, and the most effective QTL located on the same chromosome, are listed in Table 7. For example, $\mathrm{r}^{2}$ between QTL_78 and the most important SNP on chromosome 3 was 0.28 for the 50K SNP chip, and the highest $\mathrm{r}^{2}$ for the 10K SNP panel was 0.02 between QTL_402 and the most important SNP at chromosome 17. Minozzi et al. (2014) simulated 3 different traits, and they also applied RF methodology to identify SNP associated with QTL. They reported that RF was successful in identifying the main QTL. However, in agreement with the present study, RF failed to detect significant associations for small QTL effects. We simulated our data based on purely additive effects. Garcia-Magarinos et al. (2009) demonstrated that RF was one of the most powerful methods for detecting true association between marker and QTL when considering interactions among SNP.

In previous studies, RF was also successfully applied to real data sets to identify specific genes. Using RF, Li et al. (2014) confirmed the already known sheep pigmentation gene. Specifically, they identified 2 markers that are strongly associated with sheep coat pigmentation, and they concluded that RF is a powerful new approach for the exploration of genome-wide associations. Nguyen et al. (2015) also applied RF, and they found 25 important SNP in close proximity to gene locations for Parkinson's disease.

\section{CONCLUSIONS}

For both RF and GBLUP, the composition of training populations is one the most important factors affecting prediction accuracy. Optimal training populations are characterized by disease incidences that are similar to the population disease incidence, allowing the highest prediction accuracies. The effect of genetic 
architecture (number of QTL, level of LD) and of heritabilities on the accuracy of GEBV is more pronounced for RF than for GBLUP. The RF method is more precise than GBLUP for differentiating between healthy and sick animals (higher AUROC) for scenarios with larger marker density. Generally, prediction accuracies are higher when using the GBLUP methodology. Only in the scenario combining the highest heritability, the dense marker panel, and the largest number of QTL, RF performs better than GBLUP. For the low-density
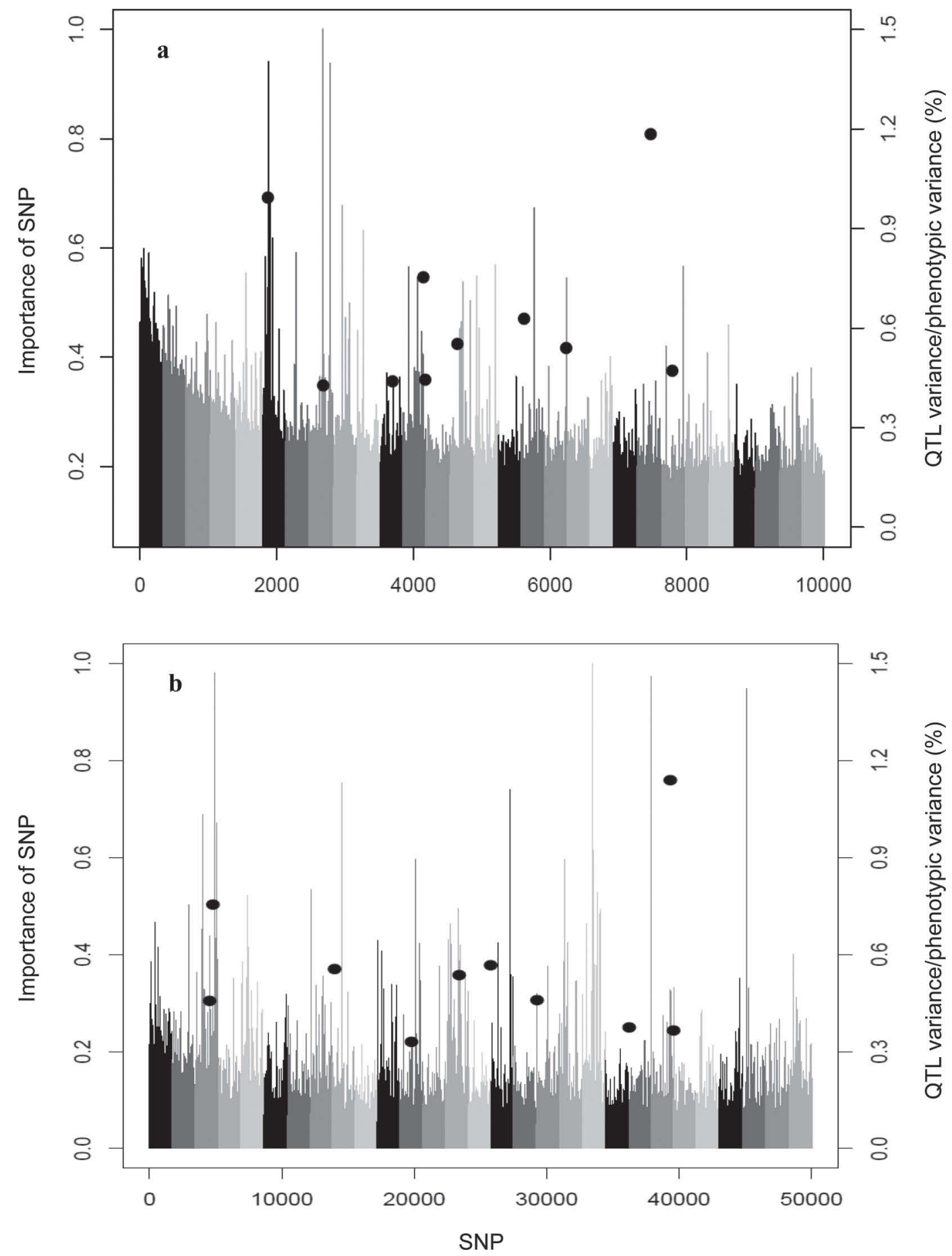

Figure 2. The relative importance of $10 \mathrm{~K}$ (a) and $50 \mathrm{~K}$ (b) SNP, and positions and percentage of phenotypic variance related to the 10 top QTL (black circles) along 29 chromosomes. 
10K SNP panel, AUROC values are quite similar for GBLUP and RF. With regard to whole-genome screenings, RF identifies important SNP in close distance to a QTL or a candidate gene. One limitation for the application of RF to large cow calibration groups is the demanding computation time.

\section{ACKNOWLEDGMENTS}

The authors gratefully acknowledge funding from the German Federal Ministry of Education and Research (BMBF) and from the Förderverein Bioökonomieforschung e.V. (FBF)/German Holstein Association (DHV), for the collaborative project "KMU-innovativ-10: Kuh$\mathrm{L}$ - cow calibration groups for the implementation of selection strategies based on high-density genotyping in dairy cattle." We thank the 2 anonymous reviewers for their detailed and specific suggestions to improve our manuscript.

\section{REFERENCES}

ADR. 2014. Annual statistics of the German Cattle Breeders Federation. Arbeitsgemeinschaft Deutscher Rinderzüchter e.V., Bonn, Germany.

Albrecht, T., V. Wimmer, H. J. Auinger, M. Erbe, C. Knaak, M. Ouzunova, H. Simianer, and C. C. Schön. 2011. Genome-based prediction of testcross values in maize. Theor. Appl. Genet. 123:339-350.

Biffani, S., C. Dimauro, N. Macciotta, A. Rossoni, A. Stella, and F. Biscarini. 2015. Predicting haplotype carriers from SNP genotypes in Bos taurus through linear discriminant analysis. Genet. Sel. Evol. 47:4

Breiman, L. 2001. Random forests. Mach. Learn. 45:5-32.

Buch, L. H., M. Kargo, P. Berg, J. Lassen, and A. C. Sørensen. 2012. The value of cows in reference populations for genomic selection of new functional traits. Animal 6:880-886.

Daetwyler, H. D., M. P. L. Calus, R. Pong-Wong, G. de Los Campos, and J. M. Hickey. 2013. Genomic prediction in animals and plants: Simulation of data, validation, reporting, and benchmarking. Genetics 193:347-365.

Daetwyler, H. D., J. M. Hickey, J. M. Henshall, S. Dominik, B. Gredler, J. H. J. van der Werf, and B. J. Hayes. 2010a. Accuracy of estimated genomic breeding values for wool and meat traits in a multi-breed sheep population. Anim. Prod. Sci. 50:1004-1010.

Daetwyler, H. D., R. Pong-Wong, B. Villanueva, and J. A. Woolliams. 2010b. The impact of genetic architecture on genome-wide evaluation methods. Genetics 185:1021-1031.

de Los Campos, G., J. M. Hickey, R. Pong-Wong, H. D. Daetwyler, and M. P. L. Calus. 2013. Whole-genome regression and prediction methods applied to plant and animal breeding. Genetics 193:327345 .

Edel, C., H. Schwarzenbacher, H. Hamann, S. Neuner, R. Emmerling, and K. U. Götz. 2011. The German-Austrian genomic evaluation system for Fleckvieh (Simmental) cattle. Interbull Bull. 44:152156.

Efron, B., and R. J. Tibshirani. 1993. An Introduction to the Bootstrap. Monographs on Statistics and Applied Probability 57. Chapman \& Hall/CRC, New York, NY.

Egger-Danner, C., A. Willam, C. Fuerst, H. Schwarzenbacher, and B. Fuerst-Waltl. 2012. Hot topic: Effect of breeding strategies using genomic information on fitness and health. J. Dairy Sci. 95:46004609

García-Magariños, M., L. U. Inaki, R. Cao, and A. Salas. 2009. Evaluating the ability of tree-based methods and logistic regression for the detection of SNP-SNP interaction. Ann. Hum. Genet. 73:360369.

Gernand, E., P. Rehbein, U. U. von Borstel, and S. König. 2012. Incidences of and genetic parameters for mastitis, claw disorders, and common health traits recorded in dairy cattle contract herds. J. Dairy Sci. 95:2144-2156.

Ghafouri-Kesbi, F., G. Rahimi-Mianji, M. Honarvar, and A. NejatiJavaremi. 2016. Predictive ability of random forest, boosting, support vector machines and genomic best linear unbiased prediction in different scenarios of genomic evaluation. Anim. Prod. Sci. $10.1071 / \mathrm{AN} 15538$.

Goddard, M. 2009. Genomic selection: Prediction of accuracy and maximisation of long term response. Genetica 136:245-257.

González-Recio, O., and S. Forni. 2011. Genome-wide prediction of discrete traits using Bayesian regressions and machine learning. Genet. Sel. Evol. 43:7.

González-Recio, O., G. J. M. Rosa, and D. Gianola. 2014. Machine learning methods and predictive ability metrics for genome-wide prediction of complex traits. Livest. Sci. 166:217-231.

Guo, Z., D. M. Tucker, C. J. Basten, H. Gandhi, E. Ersoz, B. Guo, Z. Xu, D. Wang, and G. Gay. 2014. The impact of population structure on genomic prediction in stratified populations. Theor. Appl. Genet. 127:749-762.

Hayes, B. J., P. J. Bowman, A. J. Chamberlin, and M. E. Goddard. 2009. Invited review: Genomic selection in dairy cattle: Progress and challenges. J. Dairy Sci. 92:433-443.

Hernandez, L. M., and D. G. Blazer. 2006. Genes, Behavior, and the Social Environment: Moving Beyond the Nature/Nurture Debate. National Academies Press, Washington, DC.

Hill, W. G., and A. Robertson. 1968. Linkage disequilibrium in finite populations. Theor. Appl. Genet. 38:226-231.

König, S., K. Brügemann, and E. C. G. Pimentel. 2013. Züchterische Strategien für Tier- und Klimaschutz: Was ist möglich und was brauchen wir? Zuchtungskunde 85:22-33.

König, S., G. Dietl, I. Raeder, and H. H. Swalve. 2005. Genetic relationships for dairy performance between large-scale and smallscale farm conditions. J. Dairy Sci. 88:4087-4096.

König, S., H. Simianer, and A. Willam. 2009. Economic evaluation of genomic breeding programs. J. Dairy Sci. 92:382-391.

Kramer, M., M. Erbe, F. R. Seefried, B. Gredler, B. Bapst, A. Bieber, and H. Simianer. 2014. Accuracy of direct genomic values for functional traits in Brown Swiss cattle. J. Dairy Sci. 97:1774-1781.

Li, Y., J. Kijas, M. Henshall, S. Lehnert, R. McCulloch, and A. Reverter. 2014. Using random forests $(\mathrm{RF})$ to prescreen candidate genes: A new prospective for GWAS. Abstract 206 in Proc. 10th World Congr. Genet. Appl. Livest. Prod., Vancouver, BC, Canada.

Madsen, P., and J. Jensen. 2010. A User's Guide to DMU: A Package for Analysing Multivariate Mixed Models. Version 6, release 5.0. University of Aarhus, Tjele, Denmark.

Makowsky, R., N. M. Pajewski, Y. C. Klimentidis, A. I. Vazquez, C. W. Duarte, D. B. Allison, and G. de Los Campos. 2011. Beyond missing heritability: Prediction of complex traits. PLoS Genet. 7:e1002051.

Mc Hugh, N., T. H. E. Meuwissen, A. R. Cromie, and A. K. Sonesson. 2011. Use of female information in dairy cattle genomic breeding programs. J. Dairy Sci. 94:4109-4118.

Meng, Y. A., Y. Yu, L. A. Cupples, L. A. Farrer, and K. L. Lunetta. 2009. Performance of random forest when SNPs are in linkage disequilibrium. BMC Bioinformatics 10:78.

Meuwissen, T. H., B. J. Hayes, and M. E. Goddard. 2001. Prediction of total genetic value using genome-wide dense marker maps. Genetics 157:1819-1829.

Minozzi, G., A. Pedretti, S. Biffani, E. L. Nicolazzi, and A. Stella 2014. Genome wide association analysis of the 16th QTL-MAS Workshop dataset using the Random Forest machine learning approach. BMC Proc. 8(Suppl. 5):S4.

Neves, H. H. R., R. Carvalheiro, and S. A. Queiroz. 2012. A comparison of statistical methods for genomic selection in a mice population. BMC Genet. 13:100.

Nguyen, T. T., J. Z. Huang, Q. Wu, T. Nguyen, and M. Junjie. 2015. Genome-wide association data classification and SNPs selection 
using two-stage quality-based random forests. BMC Genomics 16(Suppl 2):S5.

Ogutu, J. O., H. P. Piepho, and T. Schulz-Streeck. 2011. A comparison of random forests, boosting and support vector machines for genomic selection. BMC Proc. 5(Suppl. 3):S11.

Pimentel, E. C., M. Wensch-Dorendorf, S. König, and H. H. Swalve. 2013. Enlarging a training set for genomic selection by imputation of un-genotyped animals in populations of varying genetic architecture. Genet. Sel. Evol. 45:12.

Pryce, J. E., M. E. Goddard, H. W. Raadsma, and B. J. Hayes. 2010. Deterministic models of breeding scheme designs that incorporate genomic selection. J. Dairy Sci. 93:5455-5466.

Pszczola, M., T. Strabel, H. A. Mulder, and M. P. L. Calus. 2012. Reliability of direct genomic values for animals with different relationships within and to the reference population. J. Dairy Sci. 95:389-400.

Purcell, S., B. Neale, K. Todd-Brown, L. Thomas, M. A. R. Ferreira, D. Bender, J. Maller, P. Sklar, P. I. W. de Bakker, M. J. Daly, and P. C. Sham. 2007. PLINK: A tool set for whole-genome association and population-based linkage analyses. Am. J. Hum. Genet. $81: 559-575$.

Sargolzaei, M., and F. S. Schenkel. 2009. QMSim: A large-scale genome simulator for livestock. Bioinformatics 25:680-681.

$\mathrm{Su}, \mathrm{G}$., and P. Madsen. 2013. User's Guide for Gmatrix version 2, a program for computing genomic relationship matrix. Accessed Apr. 11, 2013. http://www.dmu.agrsci.dk/Gmatrix/Doc/.
Thomasen, J. R., A. C. Sørensen, M. S. Lund, and B. Guldbrandtsen. 2014. Adding cows to the reference population makes a small dairy population competitive. J. Dairy Sci. 97:5822-5832.

VanRaden, P. M. 2008. Efficient methods to compute genomic predictions. J. Dairy Sci. 91:4414-4423.

VanRaden, P. M., J. R. O'Connell, G. R. Wiggans, and K. A. Weigel. 2011. Genomic evaluations with many more genotypes. Genet. Sel. Evol. 43:10.

Vazquez, A. I., G. de Los Campos, Y. C. Klimentidis, G. J. M. Rosa, D. Gianola, N. Yi, and D. B. Allison. 2012. A comprehensive genetic approach for improving prediction of skin cancer risk in humans. Genetics 192:1493-1502.

Yin, T., and S. König. 2016. Genomics for phenotype prediction and management purposes. Anim. Front. 6:65-72.

Yin, T., E. C. G. Pimentel, V. König, U. Borstel, and S. König. 2014 Strategy for the simulation and analysis of longitudinal phenotypic and genomic data in the context of a temperature $\times$ humiditydependent covariate. J. Dairy Sci. 97:2444-2454.

Zhang, Z., J. Liu, X. Ding, P. Bijma, D. J. de Koning, and Q. Zhang. 2010. Best linear unbiased prediction of genomic breeding values using a trait-specific marker-derived relationship matrix. PLoS ONE 5:e12648. 